\title{
Some new optimal and suboptimal infinite families of undirected double-loop networks
}

\author{
Bao-Xing Chen ${ }^{1,2}$ J Ji-Xiang Meng ${ }^{2}$ and Wen-Jun Xiao ${ }^{3}$ \\ ${ }^{1}$ Dept. of Computer Science, Zhangzhou Teacher's College, Zhangzhou, P.R. of China \\ Email: cbaoxing@hotmail.com \\ ${ }^{2}$ College of Mathematics \& System Science, Xinjiang University, Wulumuqi, P.R. of China \\ ${ }^{3}$ Dept. of Computer Science, South China University of Technology, Guangzhou, P.R. of China
}

received Apr 3, 2006, revised Jul 16, 2006, accepted Oct 24, 2006.

Let $n, s$ be positive integers such that $2 \leq s<n$ and $s \neq \frac{n}{2}$. An undirected double-loop network $G(n ; 1, s)$ is an undirected graph $(V, E)$, where $V=\mathbb{Z}_{n}=\{0,1,2, \ldots, n-1\}$ and $E=\{(i, i+1(\bmod n)),(i, i+s(\bmod n)) \mid i \in \mathbb{Z}\}$. It is a circulant graph with $n$ nodes and degree 4 . In this paper, the sufficient and necessary conditions for a class of undirected double-loop networks to be optimal are presented. By these conditions, 6 new optimal and 5 new suboptimal infinite families of undirected double-loop networks are given.

AMS Subject Classifications: 05C12

Keywords: undirected double-loop networks, diameter, optimal, suboptimal

\section{Introduction}

Double-loop networks are popular in the design and implementation of metropolitan networks and parallel processing computer systems. They have many attractive properties like vertex symmetry, incremental extensibility, low valency, ease of implementation, etc. Some researchers are interested in the study of double-loop networks [1-22]. They mainly focus on designs of optimal double-loop networks [1], [2], [4], [9], [12] - [15], [18], [19], diameters [1]-[4], [9]-[15], [18]-[22] and routing [5], [7], [8], [17]. For more details we refer readers to [3], [16] and the references therein.

Let $n, s$ be positive integers such that $2 \leq s<n$ and $s \neq \frac{n}{2}$. The undirected double-loop network $G(n ; 1, s)$ is an undirected graph $(V, E)$, where $V=\mathbb{Z}_{n}=\{0,1,2, \ldots, n-1\}$ and $E=\{(i, i+1$ $\left.(\bmod n)),(i, i+s(\bmod n)) \mid i \in \mathbb{Z}_{n}\right\}$. It is a circulant graph with $n$ nodes and degree 4 . Let $d(i, j)$ be the length of a shortest path from node $i$ to node $j$. Let $d(n ; 1, s)$ denote the diameter of $G(n ; 1, s)$. Since $G(n ; 1, s)$ is vertex symmetric, $d(n ; 1, s)=\max \{d(i, j) \mid 0 \leq i, j<n\}=\max \{d(0, i) \mid 0 \leq i<n\}$. Let $D(n)=\min \{d(n ; 1, s) \mid 1<s<n\}$. Wong and Coppersmith [20] gave the lower bound $\frac{1}{2}(\sqrt{2 n}-3)$

\footnotetext{
${ }^{\dagger}$ This work was supported by the Natural Science Foundation of Fujian Province(No. Z0511035). 
for $D(n)$. Boesch and Wang [4] sharpened the bound by giving $l b(n)=\left\lceil\frac{\sqrt{2 n-1}-1}{2}\right\rceil$, where $\lceil x\rceil$ denotes the minimum integer $\geq x$.

If $s$ exists such that $D(n)=d(n ; 1, s)=l b(n)$, then $n, s$, and $G(n ; 1, s)$ will be called optimal. If $s$ exists such that $D(n)=d(n ; 1, s)=l b(n)+1$, then $n, s$, and $G(n ; 1, s)$ will be called suboptimal.

A set $\Theta$ of natural numbers will be called an optimal (suboptimal) family if each $n \in \Theta$ is optimal (suboptimal).

Some optimal infinite families of undirected double-loop networks were given in [2], [11], [12], [19]. And as we know, only one suboptimal infinite family of undirected double-loop networks $\left\{2 t^{2}+2 t \mid t \geq\right.$ $2\}$ has been given so far [12]. In this paper, the sufficient and necessary conditions for a class of undirected double-loop networks to be optimal are presented. And by using these conditions, we obtain 6 new optimal and 5 new suboptimal infinite families of undirected double-loop networks.

\section{Definitions and some lemmas}

Let $\mathbb{Z}$ and $\mathbb{Z}^{+}$be the set of integers and nonnegative integers respectively. Let $\lfloor x\rfloor$ denote the maximum integer $\leq x$.

Definition $2.1\left(a_{1}, a_{2}\right)$ is said to be a non-negative solution of the equation

$$
x+y s \equiv 0 \quad(\bmod n)
$$

if $a_{1}+a_{2} s \equiv 0(\bmod n), a_{1} \geq 0, a_{2} \geq 0$ and $\left(a_{1}, a_{2}\right) \neq(0,0)$.

$(u, v)$ is said to be the smallest non-negative solution of the equation (1) if $(u, v)$ is a non-negative solution of the equation (1) and the following conditions hold:

(1) if $\left(a_{1}, a_{2}\right)$ is a non-negative solution of the equation (1), then $u+v \leq a_{1}+a_{2}$.

(2) if $\left(a_{1}, a_{2}\right)$ is a non-negative solution of the equation (1), where $\left(a_{1}, a_{2}\right) \neq(u, v)$ and $u+v=a_{1}+a_{2}$, then $u>a_{1}$.

Clearly, the smallest non-negative solution of equation (1)is unique. For example, it is easy to see that $(4,1),(2,3),(0,5),(8,2),(4,6), \cdots$ are non-negative solutions of the equation $x+6 y \equiv 0(\bmod 10)$. Thus $(4,1)$ is the smallest non-negative solution of the equation $x+6 y \equiv 0(\bmod 10)$.

Definition 2.2 Let $(u, v)$ be the smallest non-negative solution of the equation $(1) .\left(-a_{1}, a_{2}\right)$ is said to be a cross solution of the equation (1) if $-a_{1}+a_{2} s \equiv 0(\bmod n), a_{1} \geq 0, a_{2} \geq 0,\left(-a_{1}, a_{2}\right) \neq(0,0)$, and the three points $\left(-a_{1}, a_{2}\right),(0,0),(u, v)$ are not on the same line. $(-a, b)$ is said to be the smallest cross solution of the equation (1) if $(-a, b)$ is a cross solution of the equation (1) and the following conditions hold:

(1) if $\left(-a_{1}, a_{2}\right)$ is a cross solution of the equation (17), then $a+b \leq a_{1}+a_{2}$.

(2) if $\left(-a_{1}, a_{2}\right)$ is a cross solution of the equation 17 , where $\left(-a_{1}, a_{2}\right) \neq(-a, b)$ and $a+b=a_{1}+a_{2}$, then $b>a_{2}$. 
Clearly, the smallest cross solution of equation (1) is unique. For example, it is easy to see that $(2,2)$ is the smallest non-negative solution of the equation $x+5 y \equiv 0(\bmod 12)$, and $(-5,1),(-3,3),(-1,5)$, $(-12,0),(-10,2),(-8,4),(-6,6),(-4,8),(-2,10), \cdots$ are cross solutions of the equation $x+5 y \equiv 0$ $(\bmod 12)$. Thus $(-1,5)$ is the smallest cross solution of the equation $x+5 y \equiv 0(\bmod 12)$.

From [11] we have the following two lemmas.

Lemma 2.3 Let $(u, v)$ be the smallest non-negative solution of the equation $(1)$ and $(-a, b)$ be the smallest cross solution of the equation (1) If $u<v$, then $a>u, a>b, b<v$ and $n=a v+b u$.

Lemma 2.4 Let $(u, v)$ be the smallest non-negative solution of the equation $(1)$ and $(-a, b)$ be the smallest cross solution of the equation (1). If $u \geq v$, then $a<u, a \leq b, v<b$ and $n=a v+b u$.

Given $G(n ; 1, s)$, we construct an infinite grid $G_{n, 1, s}$ in $\mathbb{Z}^{2}$ as D. Tzvieli did in [19], labelling each lattice point $(i, j)$ by $i+j s(\bmod n)$. Every label $m, 0 \leq m<n$, is repeated in $G_{n, 1, s}$ infinitely many times. We refer to a lattice point with label $i$ as an $i$-point. If $i+j s \equiv 0(\bmod n)$, then we call $(i, j)$ a 0 -point.

The smallest non-negative solution $(u, v)$ of the equation $(1)$ can be seen as a 0 -point in the first quadrant with $u+v$ is minimum (in case of tie, take the maximum $\mathrm{u}$ ). The smallest cross solution $(-a, b)$ of the equation (1) can be seen as a 0-point in the second quadrant with $a+b$ is minimum (in case of tie, take the maximum $b$ ).

For $G(12 ; 1,5)$, one can see that $(2,2)$ is a 0 -point in the first quadrant, and $(-1,5)$ is a 0 -point in the second quadrant(see Fig. 1).

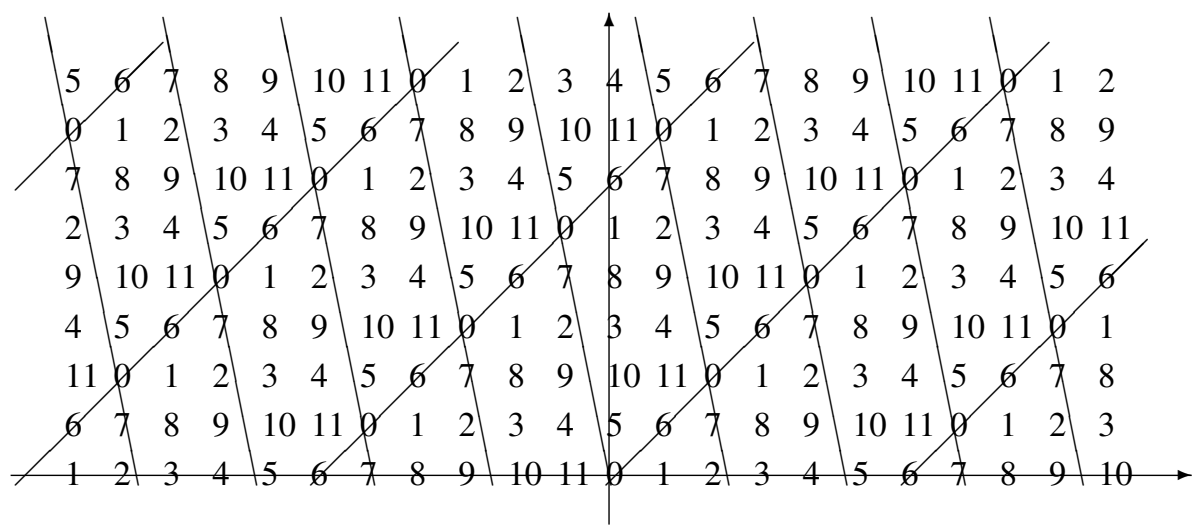

Fig. 1 0-points in the $G_{12 ; 1,5}$.

From Lemma 4 [10] and Lemma 5 [10] we have the following lemma:

Lemma 2.5 Given $G(n ; 1, s)$ with $1<s<n$ and $s \neq \frac{n}{2}$. Suppose that $(u, v)$ and $(-a, b)$ are two 0-points and $u, v, a, b$ are all non-negative integers. Let $r_{1}=\lfloor(u+v) / 2\rfloor, r_{2}=\lfloor(a+b) / 2\rfloor, r_{3}=\lfloor(\mid u-$ $a \mid+v+b) / 2\rfloor$ and $r_{4}=\lfloor(u+a+|v-b|) / 2\rfloor$. If $n=a v+b u$ and $b \geq a, u \geq v, u>a, v<b($ or $a>b, v>u, a>u, b<v)$, then

$$
d(n ; 1, s)= \begin{cases}r_{3}-1, & \text { if } r_{3}=r_{4} \text { and }(u+a)(v+b) \equiv 1 \quad(\bmod 2) \\ \max \left\{r_{1}, r_{2}, \min \left\{r_{3}, r_{4}\right\}\right\}, & \text { otherwise. }\end{cases}
$$


By Lemma 2.5, it is easy to prove the following corollary.

Corollary 2.6 Given $G(n ; 1, s)$ with $1<s<n$ and $s \neq \frac{n}{2}$. Suppose that $(u, v)$ and $(-a, b)$ are two 0 points and $u, v, a, b$ are all non-negative integers. Let d denote the diameter of $G(n ; 1, s)$. If $n=a v+b u$ and $b \geq a, u \geq v, u>a, v<b$ (or $a>b, v>u, a>u, b<v$ ), then $u+v \leq 2 d+1$ and $a+b \leq 2 d+1$.

Lemma 2.7 Suppose that $u, v, a, b$ are all integers and there exist two integers $\lambda, \chi$ such that $\lambda b+\chi v=1$. Let $s_{0} \equiv \lambda a-\chi u$ and $s=s_{0}(\bmod n)$. If $n=a v+b u$, then $u+v s \equiv 0(\bmod n)$ and $-a+b s \equiv 0$ $(\bmod n)$, i.e.,$(u, v)$ and $(-a, b)$ are two 0-points.

Proof: Since $\left(\begin{array}{cc}b & v \\ a & -u\end{array}\right)\left(\begin{array}{l}\lambda \\ \chi\end{array}\right)=\left(\begin{array}{l}1 \\ s_{0}\end{array}\right)$, we have $n\left(\begin{array}{l}\lambda \\ \chi\end{array}\right)=\left(\begin{array}{cc}u & v \\ a & -b\end{array}\right)\left(\begin{array}{l}1 \\ s_{0}\end{array}\right)$. That is, $u+v s \equiv u+v s_{0}(\bmod n)=n \lambda$ $(\bmod n)=0$ and $-a+b s \equiv-a+b s_{0}(\bmod n)=-n \chi(\bmod n)=0$.

We use $\operatorname{gcd}\left(a_{1}, a_{2}\right)$ to denote the greatest common divisor of two integers $a_{1}, a_{2}$. As the following lemma is well-known and easy to prove, its proof is omitted.

Lemma 2.8 Suppose that $a_{1}, b_{1}$ and $c_{1}$ are integers.

(1) $\operatorname{gcd}\left(a_{1}, b_{1}\right)=1$ if and only if there exist two integers $\lambda$, $\chi$ such that $\lambda a_{1}+\chi b_{1}=1$.

(2) If $\operatorname{gcd}\left(a_{1}, b_{1}\right)=1$ and $\operatorname{gcd}\left(a_{1}, c_{1}\right)=1$, then $\operatorname{gcd}\left(a_{1}, b_{1} c_{1}\right)=1$.

\section{Main results}

The sufficient and necessary conditions for a class of undirected double-loop networks to be optimal are given in the following theorem. By using this theorem, we can give some new optimal or suboptimal infinite families of undirected double-loop networks in this section.

Theorem 3.1 Suppose that $n=2 t^{2}+2 t-B$, where $t>B>0$, and $t, B \in \mathbb{Z}^{+}$. Then there exists a positive integer s such that $d(n ; 1, s)=t=l b(n)$ if and only if there exist four non-negative integers $a, b, u, v$ satisfying the following five conditions:

$1 n=a v+b u$;

$2 a+b \leq 2 t+1$ and $u+v \leq 2 t+1$;

$3 b \geq a, u \geq v, u>a, v<b$;

4 One of the following two conditions holds:

(a) $x=0, b-a=y$, $y$ is odd, $y \mid 2 B+1$ and $1 \leq y \leq \sqrt{2 B+1}$.

(b) $y=0, u-v=x, x$ is odd, $x \mid 2 B+1$ and $1 \leq x \leq \sqrt{2 B+1}$. where $x=2 t+1-(a+b)$, $y=2 t+1-(u+v)$.

$5 \operatorname{gcd}(b, v)=1$.

Proof: Suppose that there exists a positive integer $s$ such that $d(n ; 1, s)=t$. Let $(u, v)$ be the smallest non-negative solution of the equation (1) and $(-a, b)$ be the smallest cross solution of the equation (1).

In the following we consider two cases: $u \geq v$ and $u<v$. 
Case: $u \geq v \quad$ Then by Lemma 2.4 and Corollary 2.6, we see that (1), 2) and (3) hold.

Now we will prove that condition 4 holds.

Let $r_{1}=\lfloor(u+v) / 2\rfloor, r_{2}=\lfloor(a+b) / 2\rfloor, r_{3}=\lfloor(u-a+v+b) / 2\rfloor$ and $r_{4}=\lfloor(u+a+b-v) / 2\rfloor$.

If $r_{3}=r_{4}$ and $(u+a)(v+b) \equiv 1(\bmod 2)$, then as $u-a+v+b \equiv 0(\bmod 2)$ and $u+a-v+b \equiv 0$ $(\bmod 2)$, we have $u-a+v+b=u+a-v+b$, i.e., $a=v$. Thus the diameter of $G(n ; 1, s)$ is $t=(u+b) / 2-1$. So $b-a=b-v=(u+b)-(u+v)=2 t+2-(2 t+1-y)=1+y$ and $u-v=u-a=(u+b)-(a+b)=2 t+2-(2 t+1-x)=1+x$. As $(u+a)(v+b) \equiv 1(\bmod 2)$ and $a=v$, we have $x=2 t+1-(a+b)=2 t+1-(v+b) \equiv 0(\bmod 2)$ and $y=2 t+1-(u+v)=2 t+1-(u+a) \equiv 0$ $(\bmod 2)$.

As $0 \leq(b-a)(u-v)=(1+y)(u-v) \leq(1+y)(u+v)$ and $(b-a)(u-v)=2(a v+b u)-(a+b)(u+v)=$ $2 n-(2 t+1-x)(2 t+1-y)$, we have $2 n-(2 t+1-x)(2 t+1-y) \leq(1+y)(u+v)=(1+y)(2 t+1-y)$, i.e., $(2 t+2-x+y)(2 t+1-y) \geq 2 n$. Thus we have $\left(y+\frac{1-x}{2}\right)^{2}+(x-2)\left(2 t+1-\frac{x}{4}\right) \leq \frac{1}{4}+2 B-2 t$. As $\frac{1}{4}+2 B-2 t<0$ and $2 t+1-\frac{x}{4}>0$, we have $x<2$. Similarly we can prove that $y<2$. Since $x \equiv 0(\bmod 2), y \equiv 0(\bmod 2), x \geq 0$ and $y \geq 0$, we have $x=y=0$. As $b-a=1+y=1$, $u-v=1+x=1, a+b=2 t+1-x=2 t+1$ and $u+v=2 t+1-y=2 t+1$, we have $a=v=t$, $b=u=t+1$. Since $a v+b u=t^{2}+(t+1)^{2}=2 t^{2}+2 t+1$ and $n=a v+b u$, we get a contradiction. Thus $r_{3}=r_{4}$ and $(u+a)(v+b) \equiv 1(\bmod 2)$ can not hold simultaneously.

In other cases, the diameter of $G(n ; 1, s)$ is $\max \left\{r_{1}, r_{2}, \min \left\{r_{3}, r_{4}\right\}\right\}=t$. Thus $r_{3} \leq t$ or $r_{4} \leq t$.

If $r_{3} \leq t$, we have $b-a \leq y$. As $0 \leq(b-a)(u-v) \leq y(u+v)$ and $(b-a)(u-v)=2(a v+b u)-$ $(a+b)(u+v)=2 n-(2 t+1-x)(2 t+1-y)$, we have $(2 t+1-x+y)(2 t+1-y) \geq 2 n$, i.e., $4 t^{2}+(4-2 x) t+(1-x+y)(1-y) \geq 2 n$. Thus we have $\left(y-\frac{x}{2}\right)^{2}+(x-1)\left(2 t+1-\frac{x+1}{4}\right) \leq \frac{1}{4}+2 B-2 t$. As $\frac{1}{4}+2 B-2 t<0$ and $2 t+1-\frac{x+1}{4}>0$, we have $x<1$. Thus $x=0$ and $y \leq \sqrt{2 B+1}$. As $x=0$ and $0 \leq(b-a)(u-v)=-2 B-1-x y+(x+y)(2 t+1)=-2 B-1+y(2 t+1)$, we have $y \geq 1$. As $u-v=\frac{-2 B-1+y(2 t+1)}{b-a}=\frac{2 t-2 B+(y-1)(2 t+1)}{b-a} \leq u+v=2 t+1-y$, we have $b-a>y-1$. Since $b-a \leq y$, we have $b-a=y$. As $b+a=2 t+1, b-a=b+a-2 a$, we see that $y=b-a$ is odd. As $b-a=y$ and $(b-a)(u-v)=-2 B-1+y(2 t+1)$, we have $2 B+1=y(2 t+1-u+v)$. Thus $y$ divides $2 B+1$, i.e., $y \mid 2 B+1$.

If $r_{4} \leq t$, we have $u-v \leq x$. As in the case of $r_{3} \leq t$, we can prove that $y=0, u-v=x, x$ is odd, $x \mid 2 B+1$ and $1 \leq x \leq \sqrt{2 B+1}$.

From above we see that condition (4) holds.

Since $u+v s \equiv 0(\bmod n)$ and $-a+b s \equiv 0(\bmod n)$, there exist two integers $\lambda$ and $\chi$ such that $\left(\begin{array}{cc}u & v \\ -a & b\end{array}\right)\left(\begin{array}{l}1 \\ s\end{array}\right)=n\left(\begin{array}{l}\lambda \\ \chi\end{array}\right)$. Thus $\left(\begin{array}{l}1 \\ s\end{array}\right)=\left(\begin{array}{cc}b & -v \\ a & u\end{array}\right)\left(\begin{array}{l}\lambda \\ \chi\end{array}\right)$. So $\lambda b+(-\chi) v=1$. By Lemma 2.8 , we have $\operatorname{gcd}(v, b)=1$. Thus condition (5) holds.

Case: $u<v \quad$ By a similar argument we can deduce the following:

(1) $n=a v+b u$;

(2) $a+b \leq 2 t+1$ and $u+v \leq 2 t+1$;

(3) $a>b, v>u, a>u, b<v$;

(4) One of the following two conditions holds:

(a) $x=0, a-b=y, y$ is odd, $y \mid 2 B+1$ and $1 \leq y \leq \sqrt{2 B+1}$. 
(b) $y=0, v-u=x, x$ is odd, $x \mid 2 B+1$ and $1 \leq x \leq \sqrt{2 B+1}$. where $x=2 t+1-(a+b)$, $y=2 t+1-(u+v)$.

(5) $\operatorname{gcd}(b, v)=1$.

By letting $a^{\prime}=u, b^{\prime}=v, u^{\prime}=a, v^{\prime}=b, x^{\prime}=2 t+1-\left(a^{\prime}+b^{\prime}\right)=y$ and $y^{\prime}=2 t+1-\left(u^{\prime}+v^{\prime}\right)=x$, it is routine to verify that $a^{\prime}, b^{\prime}, u^{\prime}$ and $v^{\prime}$ satisfy the five conditions of the theorem.

On the other hand, if there exist four non-negative integers $a, b, u, v$ satisfying the five conditions of the theorem, then by condition 5 there exist two integers $\lambda$ and $\chi$ such that $\lambda b+\chi v=1$. Let $s \equiv \lambda a-\chi u$ $(\bmod n)$. By Lemma 2.7, we see that $(u, v)$ and $(-a, b)$ are two 0 -points.

As $u \geq v, b \geq a, u>a$, and $v<b$, by Lemma 2.5 we have $d(n ; 1, s) \leq t$. Since $d(n ; 1, s) \geq l b(n)=t$, we have $d(n ; 1, s)=t$.

Corollary 3.2 Suppose that $n=2 t^{2}+2 t-B$, where $t>B>0, t, B \in \mathbb{Z}^{+}$and $2 B+1$ is a prime number. Then $n$ is optimal if and only if $\operatorname{gcd}(t, B)=1$ or $\operatorname{gcd}(t+1, B)=1$.

Proof: Suppose that $n$ is optimal, by Theorem 3.1 there exist four non-negative integers $a, b, u, v$ satisfying the condition 4 of Theorem 3.1 Let $x=2 t+1-(a+b)$ and $y=2 t+1-(u+v)$. Since $2 B+1$ is a prime number, we have (a) $x=0, b-a=y=1$ or (b) $y=0, u-v=x=1$.

When $x=0, b-a=y=1$, as $(b-a)(u-v)=2(a v+b u)-(a+b)(u+v)=2 n-(2 t+1) * 2 t=2 t-2 B$, we have $a=t, b=t+1, u=2 t-B$ and $v=B$. Since $a, b, u, v$ satisfy the condition 5 of Theorem 3.1 . we have $1=\operatorname{gcd}(b, v)=\operatorname{gcd}(t+1, B)$.

When $y=0, u-v=x=1$, as $(b-a)(u-v)=2(a v+b u)-(a+b)(u+v)=2 n-2 t *(2 t+1)=$ $2 t-2 B$, we have $a=B, b=2 t-B, u=t+1$ and $v=t$. Since $a, b, u, v$ satisfy the condition 5 of Theorem 3.1. we have $1=\operatorname{gcd}(b, v)=\operatorname{gcd}(2 t-B, t)=\operatorname{gcd}(-B, t)=\operatorname{gcd}(t, B)$.

Thus we have proved that if $n$ is optimal, then $\operatorname{gcd}(t, B)=1$ or $\operatorname{gcd}(t+1, B)=1$.

If $\operatorname{gcd}(t, B)=1$ or $\operatorname{gcd}(t+1, B)=1$, we will prove that $n$ is optimal.

When $\operatorname{gcd}(t, B)=1$, let $a=B, b=2 t-B, u=t+1$ and $v=t$. It is routine to verify that four non-negative integers $a, b, u, v$ satisfy the five conditions of Theorem 3.1 .

When $\operatorname{gcd}(t+1, B)=1$, let $a=t, b=t+1, u=2 t-B$ and $v=B$. It is easy to see that four non-negative integers $a, b, u, v$ satisfy the five conditions of Theorem 3.1 .

From above we see that Corollary 3.2 holds.

By Theorem 3.1 and Corollary 3.2 , we can give some new optimal and suboptimal infinite families of undirected double-loop networks in the following theorems.

Theorem 3.3 (1) Let $\Theta=\left\{2 t^{2}+2 t-6 \mid t>6, t \neq 6 e+2\right.$ and $t \neq 6 e+3$, $\left.e \in \mathbb{Z}^{+}\right\}$. Then $\Theta$ is an optimal infinite family, and when $t=6 e, 6 e+1,6 e+4,6 e+5$ respectively, the optimal step $s$ is $12 e^{2}, 12 e^{2}+4 e-2,60 e^{2}+88 e+28,60 e^{2}+108 e+42$ correspondingly.

(2) $\left\{G\left(2 t^{2}+2 t-6 ; 1,2 t^{2}-3\right) \mid t=6 e+2\right.$ or $\left.t=6 e+3,1 \leq e \in \mathbb{Z}^{+}\right\}$is a suboptimal infinite family.

\section{Proof:}

(1) Let $n$ be $2 t^{2}+2 t-6$. In the following we consider two cases: $t=6 e+1$ or $t=6 e+5$ and $t=6 e$ or $t=6 e+4$. 
$t=6 e+1$ or $t=6 e+5 \quad$ When $t=6 e+1$ or $t=6 e+5$, we have $\operatorname{gcd}(t, 6)=1$.

$t=6 e$ or $t=6 e+4$ When $t=6 e$ or $t=6 e+4$, we have $\operatorname{gcd}(t+1,6)=1$.

As $2 \times 6+1=13$ and 13 is a prime number, by Corollary 3.2, we see that $\Theta$ is an optimal infinite family.

When $t=6 e+1$, let $a=6, b=2 t-6, u=t+1$, and $v=t$. As $e \times b+(-2 e+1) \times v=1$, the optimal step $s$ is $e \times a-(-2 e+1) \times u(\bmod n)=12 e^{2}+4 e-2$.

When $t=6 e+5$, let $a=6, b=2 t-6, u=t+1$, and $v=t$. As $-(e+1) \times b+(2 e+1) \times v=1$, $s=-(e+1) \times a-(2 e+1) \times u(\bmod n)=60 e^{2}+108 e+42$.

When $t=6 e$, let $a=t, b=t+1, u=2 t-6$, and $v=6$. As $1 \times b-e \times v=1, s=1 \times a-(-e) \times u$ $(\bmod n)=12 e^{2}$.

When $t=6 e+4$, let $a=t, b=t+1, u=2 t-6$, and $v=6$. As $-1 \times b+(e+1) \times v=1$, $s=-1 \times a-(e+1) \times u(\bmod n)=60 e^{2}+88 e+28$.

(2) When $t=6 e+2$, we have $\operatorname{gcd}(t+1,6)=\operatorname{gcd}(6 e+3,6)=\operatorname{gcd}(3,6)=3$ and $\operatorname{gcd}(t, 6)=$ $\operatorname{gcd}(6 e+2,6)=\operatorname{gcd}(2,6)=2$.

When $t=6 e+3$, we have $\operatorname{gcd}(t+1,6)=\operatorname{gcd}(6 e+4,6)=\operatorname{gcd}(4,6)=2$ and $\operatorname{gcd}(t, 6)=$ $\operatorname{gcd}(6 e+3,6)=\operatorname{gcd}(3,6)=3$.

As $2 \times 6+1=13$ and 13 is a prime number, by Corollary 3.2 , we see that when $t=6 e+2$ or $t=6 e+3,1 \leq e \in \mathbb{Z}^{+}, n=2 t^{2}+2 t-6$ can not be optimal.

Let $a=t, b=t+2, u=2 t-3$ and $v=1$. As $0 \times b+1 \times v=1$, let $s=0 \times a-1 \times u \equiv 2 t^{2}-3$ $(\bmod n)$. By Lemma 2.7 we see that $(2 t-3,1)$ and $(-t, t+2)$ are two 0 -points. By Lemma 2.5 we have $d(n ; 1, s)=t+1$.

From above we see that when $t=6 e+2$ or $t=6 e+3,1 \leq e \in \mathbb{Z}^{+}, G\left(2 t^{2}+2 t-6 ; 1,2 t^{2}-3\right)$ is suboptimal.

Theorem 3.4 1. Let $n=2 t^{2}+2 t-12$ and $\Theta=\left\{2 t^{2}+2 t-12 \mid t>12, t \neq 6 e+2\right.$ and $t \neq 6 e+3$, $\left.e \in \mathbb{Z}^{+}\right\}$. Then $\Theta$ is an optimal infinite family, and when $t=6 e, 6 e+1,6 e+4,6 e+5$ respectively, the optimal step s is $6 e-36 e^{3}(\bmod n), 36 e^{3}+24 e^{2}-2 e-2(\bmod n),-36 e^{3}-96 e^{2}-78 e-16$ $(\bmod n), 36 e^{3}+96 e^{2}+78 e+18(\bmod n)$ correspondingly.

2. Let $\Psi=\left\{2 t^{2}+2 t-12 \mid t=6 e+2\right.$ or $t=6 e+3$, where $e \in \mathbb{Z}^{+}$and $\left.e \geq 2\right\}$. Then $\Psi$ is a suboptimal infinite family, and when $t=6 e+2$ or $6 e+3$, the suboptimal step $s$ is $t^{2}+2 t-3, t^{2}-4$ correspondingly.

\section{Proof:}

(1) By Theorem 3.1, we only need to find four non-negative integers $a, b, u, v$ which satisfy the five conditions of Theorem 3.1. In the following we consider two cases: $t=6 e$ or $t=6 e+4$, and $t=6 e+1$ or $t=6 e+5$.

$t=6 e$ or $t=6 e+4 \quad$ When $t=6 e$ or $t=6 e+4$, let $a=t, b=t+1, u=2 t-12$ and $v=12$.

When $t=6 e$ or $t=6 e+4, \operatorname{As} \operatorname{gcd}(2, t+1)=1$ and $\operatorname{gcd}(3, t+1)=1$, by Lemma 2.8, we have that $\operatorname{gcd}(4, t+1)=1$ and $\operatorname{gcd}(12, t+1)=1$. That is, $\operatorname{gcd}(v, b)=1$. Thus it is easy to verify that four integers $a, b, u, v$ satisfy the five conditions of Theorem 3.1 
$t=6 e+1$ or $t=6 e+5 \quad$ When $t=6 e+1$ or $t=6 e+5$, let $a=12, b=2 t-12, u=t+1$ and $v=t$. When $t=6 e+1$ or $t=6 e+5$, As $\operatorname{gcd}(2, t)=1$ and $\operatorname{gcd}(3, t)=1$, by Lemma 2.8, we have that $\operatorname{gcd}(4, t)=1$ and $\operatorname{gcd}(12, t)=1$. Thus $\operatorname{gcd}(v, b)=\operatorname{gcd}(t, 2 t-12)=\operatorname{gcd}(t,-12)=\operatorname{gcd}(t, 12)=1$.

So it is easy to verify that four integers $a, b, u, v$ satisfy the five conditions of Theorem 3.1 .

From above we see that $\Theta$ is an optimal infinite family.

When $t=6 e$, let $a=t, b=t+1, u=2 t-12$, and $v=12$. As $(1-6 e) \times b+3 e^{2} \times v=1$, $s=(1-6 e) \times a-3 e^{2} \times u(\bmod n)=6 e-36 e^{3}(\bmod n)$.

When $t=6 e+1$ or $6 e+4$ or $6 e+5$, the corresponding optimal step $s$ can be computed similarly. They are $36 e^{3}+24 e^{2}-2 e-2(\bmod n),-36 e^{3}-96 e^{2}-78 e-16(\bmod n), 36 e^{3}+96 e^{2}+78 e+18$ $(\bmod n)$ respectively.

(2) For $n=2 t^{2}+2 t-12$, where $t=6 e+2$ or $t=6 e+3,2 \leq e \in \mathbb{Z}^{+}$, in the following we will prove that $n$ can not be optimal.

If $n$ is optimal, then there exist four non-negative integers $a, b, u, v$ which satisfy the five conditions of Theorem 3.1 Let $x=2 t+1-(a+b)$ and $y=2 t+1-(u+v)$.

In the following we consider two cases:

(A) $x=0, b-a=y, y$ is odd, $y \mid 2 B+1$ and $1 \leq y \leq \sqrt{2 B+1}$.

(B) $y=0, u-v=x, x$ is odd, $x \mid 2 B+1$ and $1 \leq x \leq \sqrt{2 B+1}$, where $B=12$.

Case $\mathbf{A}$ If $x=0, b-a=y, y$ is odd, $y \mid 25$ and $1 \leq y \leq \sqrt{25}$, then $y=1$ or $y=5$.

Subcase: $x=0, y=1$ : As $b-a=y$ and $(b-a)(u-v)=2 n-(a+b)(u+v)=2 n-(2 t+1-x)(2 t+$ $1-y)=2 t-24$, we have that $b-a=1$ and $u-v=2 t-24$. Thus $a=t, b=t+1, u=2 t-12$ and $v=12$. When $t=6 e+2$ or $t=6 e+3, \operatorname{gcd}(b, v) \neq 1$.

Subcase: $x=0, y=5:$ As $b-a=y$ and $(b-a)(u-v)=2 n-(a+b)(u+v)=2 n-(2 t+1-x)(2 t+$ $1-y)=10 t-20$, we have that $b-a=5$ and $u-v=2 t-4$. Thus $a=t-2, b=t+3, u=2 t-4$ and $v=0$. So $\operatorname{gcd}(b, v)=t+3 \neq 1$.

Case $\mathbf{B}$ If $y=0, u-v=x, x$ is odd, $x \mid 25$ and $1 \leq x \leq \sqrt{25}$, then $x=1$ or $x=5$.

Subcase: $y=0, x=1$ : As $u-v=x$ and $(b-a)(u-v)=2 n-(a+b)(u+v)=2 n-(2 t+1-x)(2 t+$ $1-y)=2 t-24$, we have $u-v=1$ and $b-a=2 t-24$. Thus $a=12, b=2 t-12, u=t+1$ and $v=t$. When $t=6 e+2$ or $t=6 e+3, \operatorname{gcd}(b, v) \neq 1$.

Subcase: $y=0, x=5$ : As $u-v=x$ and $(b-a)(u-v)=2 n-(a+b)(u+v)=2 n-(2 t+1-x)(2 t+$ $1-y)=10 t-20$, we have that $u-v=5$ and $b-a=2 t-4$. Thus $a=0, b=2 t-4, u=t+3$ and $v=t-2$. So $\operatorname{gcd}(b, v)=\operatorname{gcd}(2 t-4, t-2)=t-2 \neq 1$.

From above we see that in any cases, two non-negative integers $b, v$ can not satisfy condition 5 of Theorem 3.1 So $n$ can not be optimal.

When $t=6 e+2$, let $a=0, b=2 t-4, u=t+3$ and $v=t-3$. As $\frac{4-t}{2} \times b+(t-3) \times v=1$, let $s=\frac{4-t}{2} \times a-(t-3) \times u \equiv 9-t^{2}(\bmod n)=t^{2}+2 t-3$. By Lemma 2.7 we see that $(t+3, t-3)$ and $(0,2 t-4)$ are two 0-points. By Lemma 2.5 we have $d(n ; 1, s)=t+1$. 
When $t=6 e+3$, let $a=t-2, b=t+2, u=2 t-4$ and $v=2$. As $1 \times b-\frac{t+1}{2} \times v=1$, let $s=1 \times a+\frac{t+1}{2} \times u(\bmod n)=t^{2}-4$. By Lemma 2.7. we see that $(2 t-4,2)$ and $(-t+2, t+2)$ are two 0 -points. By Lemma 2.5 we have $d(n ; 1, s)=t+1$.

Thus when $t=6 e+2$ or $t=6 e+3,2 \leq e \in \mathbb{Z}^{+}, 2 t^{2}+2 t-12$ is suboptimal.

Theorem 3.5 Let $\Theta=\left\{2 t^{2}+2 t-\left(2 A^{2}+2 A-2\right) \mid A>0, A \in \mathbb{Z}^{+}, t \geq \frac{A^{2}+A}{2}\right\}$. Then $\Theta$ is an optimal infinite family. When $t+A$ is odd, the optimal step $s$ is $t^{2}-A^{2}+1$. When $t+A$ is even, the optimal step $s$ is $t^{2}-A^{2}-2 A$.

Proof: Let $n=2 t^{2}+2 t-\left(2 A^{2}+2 A-2\right)$. When $A>0, A \in \mathbb{Z}^{+}, t \geq \frac{A^{2}+A}{2}, l b(n)=t$.

In the following we consider two cases:

$t+A$ is an odd number When $t+A$ is odd, suppose that $t+A=2 j+1$. Let $a=t-A+1, b=t+A, u=$ $2 t-2 A$ and $v=2$. As $1 \times b-j v=1$, let $s \equiv 1 \times(t-A+1)+j(2 t-2 A)(\bmod n)=t^{2}-A^{2}+1$. By Lemma 2.7, we see that $(u, v)$ and $(-a, b)$ are two 0-points. By Lemma 2.5 we have $d(n ; 1, s)=t$.

$t+A$ is an even number When $t+A$ is even, let $a=2, b=2 t-2 A, u=t+A$ and $v=t-A+1$. Since $\frac{t-A}{2} b+(1-t+A) v=1$, let $s \equiv \frac{t-A}{2} a-(1-t+A) * u(\bmod n)=t^{2}-A^{2}-2 A$. By Lemma 2.7 we see that $(u, v)$ and $(-a, b)$ are two 0-points. By Lemma 2.5 we have $d(n ; 1, s)=t$.

Thus when $t \geq \frac{A^{2}+A}{2}, 0<A \in \mathbb{Z}^{+}, 2 t^{2}+2 t-\left(2 A^{2}+2 A-2\right)$ is optimal.

By Theorem 3.5, it is easy to prove the following corollary.

Corollary 3.6 Let $\Gamma=\left\{2 t^{2}-2 \mid t=A^{2}+A-2, A \geq 2, A \in \mathbb{Z}^{+}\right\}$. Then $\Gamma$ is an optimal infinite family. When $A$ is odd, the optimal step $s$ is $t^{2}-A^{2}+1$. When $A$ is even, the optimal step $s$ is $t^{2}-A^{2}-2 A$.

By APPENDIX B [19], we see that $390=2 \times 14^{2}-2$ is suboptimal. In other words, there are some suboptimal integers in the set $\Sigma=\left\{2 t^{2}-2 \mid t>2, t \in \mathbb{Z}^{+}\right\}$. From Corollary 3.6, we see that the set $\Gamma$, which is a subset of the set $\Sigma$, is optimal. It would be interesting to find out a subset of the set $\Sigma$ which is a suboptimal infinite family.

Theorem 3.7 (1) Let $n=2 t^{2}+2 t-14$ and $\Theta=\left\{2 t^{2}+2 t-14 \mid t>14, t \neq 14 e+6\right.$ and $t \neq 14 e+7$, $\left.e \in \mathbb{Z}^{+}\right\}$. Then $\Theta$ is an optimal infinite family, and when $t=14 e, 14 e+1,14 e+2,14 e+3,14 e+$ $4,14 e+5,14 e+8,14 e+9,14 e+10,14 e+11,14 e+12,14 e+13$ respectively, the optimal step sis $28 e^{2}, 28 e^{2}+4 e-2,140 e^{2}+48 e, 140 e^{2}+68 e+2,84 e^{2}+52 e+6,84 e^{2}+64 e+8,-84 e^{2}-104 e-$ $28(\bmod n),-84 e^{2}-116 e-38(\bmod n),-140 e^{2}-212 e-74(\bmod n),-140 e^{2}-232 e-92$ $(\bmod n),-28 e^{2}-52 e-22(\bmod n),-28 e^{2}-56 e-28(\bmod n)$ correspondingly.

(2) Let $\Psi=\left\{2 t^{2}+2 t-14 \mid t=14 e+6\right.$ or $t=14 e+7$, where $e \in \mathbb{Z}^{+}$and $\left.e \geq 1\right\}$. Then $\Psi$ is a suboptimal infinite family, and when $t=14 e+6$ or $14 e+7$, the suboptimal step s is $t^{2}+2 t-4, t^{2}-5$ correspondingly.

Proof:

(1) In the following we consider two cases:

(A) $t=14 e$, or $t=14 e+2$, or $t=14 e+4$, or $t=14 e+8$, or $t=14 e+10$, or $t=14 e+12$;

(B) $t=14 e+1$, or $t=14 e+3$, or $t=14 e+5$, or $t=14 e+9$, or $t=14 e+11$, or $t=14 e+13$. 
Case $\mathbf{A}$ When $t=14 e$, or $t=14 e+2$, or $t=14 e+4$, or $t=14 e+8$, or $t=14 e+10$, or $t=14 e+12$, we have $\operatorname{gcd}(t+1,14)=1$.

Case B When $t=14 e+1$, or $t=14 e+3$, or $t=14 e+5$, or $t=14 e+9$, or $t=14 e+11$, or $t=14 e+13$, we have $\operatorname{gcd}(t, 14)=1$.

As $2 \times 14+1=29$ and 29 is a prime number, by Corollary 3.2, we see that $\Theta$ is an optimal infinite family.

When $t=14 e$, let $a=t, b=t+1, u=2 t-14$, and $v=14$. As $1 \times b-e \times v=1, s=1 \times a+e \times u$ $(\bmod n)=28 e^{2}$.

When $t=14 e+1$ or $14 e+2$ or $14 e+3$ or $14 e+4$ or $14 e+5$ or $14 e+8$ or $14 e+9$ or $14 e+10$ or $14 e+11$ or $14 e+12$ or $14 e+13$, the corresponding optimal step $s$ can be computed similarly. They are $28 e^{2}+4 e-2,140 e^{2}+48 e, 140 e^{2}+68 e+2,84 e^{2}+52 e+6,84 e^{2}+64 e+8,-84 e^{2}-$ $104 e-28(\bmod n),-84 e^{2}-116 e-38(\bmod n),-140 e^{2}-212 e-74(\bmod n),-140 e^{2}-232 e-92$ $(\bmod n),-28 e^{2}-52 e-22(\bmod n),-28 e^{2}-56 e-28(\bmod n)$ respectively.

(2) When $t=14 e+6$, we have $\operatorname{gcd}(t, 14)=\operatorname{gcd}(14 e+6,14)=\operatorname{gcd}(6,14)=2$ and $\operatorname{gcd}(t+1,14)=$ $\operatorname{gcd}(14 e+7,14)=\operatorname{gcd}(7,14)=7$.

When $t=14 e+7$, we have $\operatorname{gcd}(t, 14)=\operatorname{gcd}(14 e+7,14)=7$ and $\operatorname{gcd}(t+1,14)=\operatorname{gcd}(14 e+8,14)=$ $\operatorname{gcd}(8,14)=2$.

As $2 \times 14+1=29$ and 29 is a prime number, by Corollary 3.2 we see that when $t=14 e+6$ or $t=14 e+7,1 \leq e \in \mathbb{Z}^{+}, n=2 t^{2}+2 t-14$ can not be optimal.

When $t=14 e+6$, let $a=2, b=2 t-4, u=t+2$ and $v=t-3$. As $\frac{4-t}{2} \times b+(t-3) \times v=1$, let $s=\frac{4-t}{2} \times a-(t-3) \times u \equiv 10-t^{2}(\bmod n)=t^{2}+2 t-4$. By Lemma 2.7. we see that $(t+2, t-3)$ and $(-2,2 t-4)$ are two 0 -points. By Lemma 2.5, we have $d(n ; 1, s)=t+1$.

When $t=14 e+7$, let $a=t-3, b=t+2, u=2 t-4$ and $v=2$. As $1 \times b-\frac{t+1}{2} \times v=1$, let $s \equiv 1 \times a+\frac{t+1}{2} \times u(\bmod n)=t^{2}-5$. By Lemma 2.7. we see that $(2 t-4,2)$ and $(-t+3, t+2)$ are two 0 -points. Thus by Lemma 2.5 we have $d(n ; 1, s)=t+1$.

From above we see that when $t=14 e+6$ or $t=14 e+7$, where $1 \leq e \in \mathbb{Z}^{+}, 2 t^{2}+2 t-14$ is suboptimal.

Theorem 3.8 1. Let $\Theta=\left\{2 t^{2}+2 t-15 \mid t>15, t \neq 15 e+5\right.$ and $t \neq 15 e+9$, $\left.e \in \mathbb{Z}^{+}\right\}$. Then $\Theta$ is an optimal infinite family, and when $t=15 e, 15 e+1,15 e+2,15 e+3,15 e+4,15 e+6,15 e+$ $7,15 e+8,15 e+10,15 e+11,15 e+12,15 e+13,15 e+14$ respectively, the optimal step $s$ is $30 e^{2}, 240 e^{2}+46 e-5,240 e^{2}+78 e-3,120 e^{2}+54 e+3,120 e^{2}+70 e+5,-60 e^{2}-54 e-9$ $(\bmod n), 60 e^{2}+58 e+13,60 e^{2}+66 e+15,330 e^{2}+460 e+150,330 e^{2}+504 e+180,210 e^{2}+$ $348 e+138,-30 e^{2}-56 e-24(\bmod n),-30 e^{2}-60 e-30(\bmod n)$ correspondingly.

2. $\left\{G\left(2 t^{2}+2 t-15 ; 1,2 t^{2}-10\right) \mid t=15 e+5\right.$ or $t=15 e+9$, where $\left.1 \leq e \in \mathbb{Z}^{+}\right\}$is a suboptimal infinite family.

\section{Proof:}

(1) In the following we consider two cases:

(A) $t=15 e$, or $t=15 e+1$, or $t=15 e+3$, or $t=15 e+6$, or $t=15 e+7$, or $t=15 e+10$, or $t=15 e+12$, or $t=15 e+13$; 
(B) $t=15 e+2$, or $t=15 e+4$, or $t=15 e+8$, or $t=15 e+11$, or $t=15 e+14$.

Case $\mathbf{A}$ When $t=15 e$, or $t=15 e+1$, or $t=15 e+3$, or $t=15 e+6$, or $t=15 e+7$, or $t=15 e+10$, or $t=15 e+12$, or $t=15 e+13$, we have $\operatorname{gcd}(t+1,15)=1$.

Case B When $t=15 e+2$, or $t=15 e+4$, or $t=15 e+8$, or $t=15 e+11$, or $t=15 e+14$, we have $\operatorname{gcd}(t, 15)=1$.

As $2 \times 15+1=31$ and 31 is a prime number, by Corollary 3.2 , we see that $\Theta$ is an optimal infinite family.

When $t=15 e$, let $a=t, b=t+1, u=2 t-15$, and $v=15$. As $1 \times b-e \times v=1, s=1 \times a+e \times u$ $(\bmod n)=30 e^{2}$.

When $t=15 e+1$ or $15 e+2$ or $15+3$ or $15 e+4$ or $15 e+6$ or $15 e+7$ or $15 e+8$ or $15 e+10$ or $15 e+11$ or $15 e+12$ or $15 e+13$ or $15 e+14$, the corresponding optimal step $s$ can be computed similarly. They are $240 e^{2}+46 e-5,240 e^{2}+78 e-3,120 e^{2}+54 e+3,120 e^{2}+70 e+5,-60 e^{2}-54 e-9$ $(\bmod n), 60 e^{2}+58 e+13,60 e^{2}+66 e+15,330 e^{2}+460 e+150,330 e^{2}+504 e+180,210 e^{2}+348 e+$ $138,-30 e^{2}-56 e-24(\bmod n),-30 e^{2}-60 e-30(\bmod n)$ respectively.

(2) When $t=15 e+5$, we have $\operatorname{gcd}(t, 15)=\operatorname{gcd}(15 e+5,15)=\operatorname{gcd}(5,15)=5$ and $\operatorname{gcd}(t+1,15)=$ $\operatorname{gcd}(15 e+6,15)=\operatorname{gcd}(6,15)=3$.

When $t=15 e+9$, we have $\operatorname{gcd}(t, 15)=\operatorname{gcd}(15 e+9,15)=\operatorname{gcd}(9,15)=3$ and $\operatorname{gcd}(t+1,15)=$ $\operatorname{gcd}(15 e+10,15)=\operatorname{gcd}(10,15)=5$.

As $2 \times 15+1=31$ and 31 is a prime number, by Corollary 3.2 , we see that when $t=15 e+5$ or $t=15 e+9,1 \leq e \in \mathbb{Z}^{+}, n=2 t^{2}+2 t-15$ can not be optimal.

When $t=15 e+5$ or $t=15 e+9$, let $a=t, b=t+3, u=2 t-5$ and $v=1$. As $0 \times b+1 \times v=1$, let $s=0 \times a-1 \times u \equiv 5-2 t(\bmod n)=2 t^{2}-10$. By Lemma 2.7, we see that $(2 t-5,1)$ and $(-t, t+3)$ are two 0-points. By Lemma 2.5, we have $d(n ; 1, s)=t+1$.

From above we see that when $t=15 e+5$ or $t=15 e+9$, where $1 \leq e \in \mathbb{Z}^{+}, G\left(2 t^{2}+2 t-15 ; 1,2 t^{2}-\right.$ 10) is suboptimal.

\section{Acknowledgements}

The authors thank the referees for their helpful comments that improved the accuracy and clarity of our presentation.

\section{References}

[1] F. Aguiló and M. A. Fiol, An efficient algorithm to find optimal double loop networks, Discrete Mathematics 138(1995), 15-29.

[2] J. -C. Bermond and D. Tzvieli, Minimal diameter double-loop networks: dense optimal families, Networks 21(1991), 1-9.

[3] J. -C. Bermond, F. Comellas and D. F. Hsu, Distributed loop computer networks: a survey, Journal of Parallel and Distributed Computing 24(1995), 2-10.

[4] F. T. Boesch and J. F. Wang, Reliable circulant networks with minimum transmission delay, IEEE Trans. Circuits Syst. CAS-32(1985), 1286-1291. 
[5] N. Chalamaiah and B. Ramamurty, Finding shortest paths in distributed loop networks, Information Processing Letters 67(1998), 157-161.

[6] R. C.-F. Chan, C.-Y. Chen, Z.-X. Hong, A simple algorithm to find the steps of double-loop networks, Discrete Applied Mathematics 121(2002), 61-72.

[7] B. X. Chen, J. X. Meng and W. J. Xiao, A constant time optimal routing algorithm for undirected double-loop networks, International Conference on Mobile Ad-hoc and Sensor Networks, Wuhan, China, Lecture Notes in Computer Science (LNCS), Springer Verlag , 3794(2005), 308-316.

[8] B. X. Chen and W. J. Xiao, A constant time optimal routing algorithm for directed double loop networks $G\left(n ; s_{1}, s_{2}\right)$, In the proceeding of 5th International Conference on Software Engineering, Artificial Intelligence, Networking, and Parallel/Distributed Computing(SNPD 2004), 1-5.

[9] B. X. Chen and W. J. Xiao, Optimal designs of directed double-loop networks, International Symposium on Computational and Information Sciences (CIS'04), Lecture Notes in Computer Science (LNCS), Springer Verlag, 3314(2004), 19-24.

[10] B. X. Chen and W. J. Xiao, B. Parhami, Diameter Formulas for a Class of Undirected Double-loop networks, Journal of Interconnection Networks 6(2005), 1: 1-15.

[11] B. X. Chen and W. J. Xiao, A diameter formula for an undirected double-loop network, Accepted by Ars Combinatoria.

[12] D. Z. Du, D. F. Hsu, Li Qiao and Xu Jun-ming, A combinatorial problem related to distributed loop networks, Networks 20(1990), 173-180.

[13] P. Esqué, F. Aguiló and M. A. Fiol, Double commutative-step digraphs with minimum diameters, Discrete Mathematics 114(1993), 147-157.

[14] D. F. Hsu and J. Shapiro, Bounds for the minimal number of transmission delays in double loop networks, Journal of Combinatorics, Information \& System Sciences 16(1991), 55-62.

[15] F. K. Hwang and Y. H. Xu, Double loop networks with minimum delay, Discrete Mathematics 66(1987), 109-118.

[16] F. K. Hwang, A complementary survey on double-loop networks, Theoretical Computer Science 263(2001), 211-229.

[17] K. Mukhopadhyaya and B. P. Sinha, Fault-tolerant routing in distributed loop networks, IEEE Transactions on Computers 44(1995), 12:1452-1456.

[18] Q. Li, J. M. Xu and Z. L. Zhang, Infinite families of optimal double loop networks, Science in China, Ser A 23(1993), 979-992.

[19] D. Tzvieli, Minimal diameter double-loop networks I. Large infinite Optimal families, Networks 21(1991), 387-415.

[20] C. K. Wong and D. Coppersmith, A combinatorial problem related to multimodule memory organizations, Journal of the Association for Computing Machinery 21(1974), 3:392-402. 
[21] J. A. L. Yebra, M. A. Fiol, P. Morillo and I. Alegre, The diameter of undirected graphs associated to plane tessellations, Ars Combinatoria 20-B(1985), 151-171.

[22] J. Zerovnik and T. Pisanski, Computing the diameter in multi-loop networks, Journal of Algorithm 14(1993), 226-243. 
